$$
\text { DOE/ER/25019-5 }
$$

\title{
Department of Applied Mathematics
}

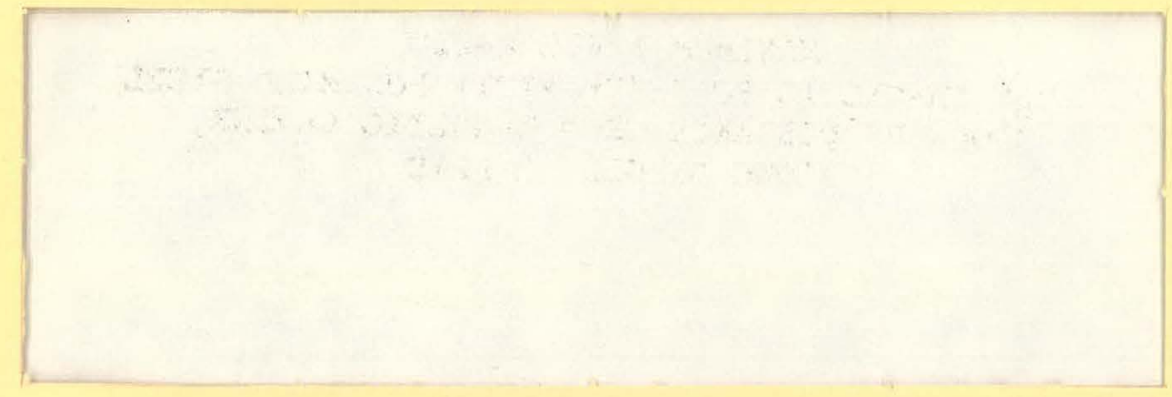

University of Washington

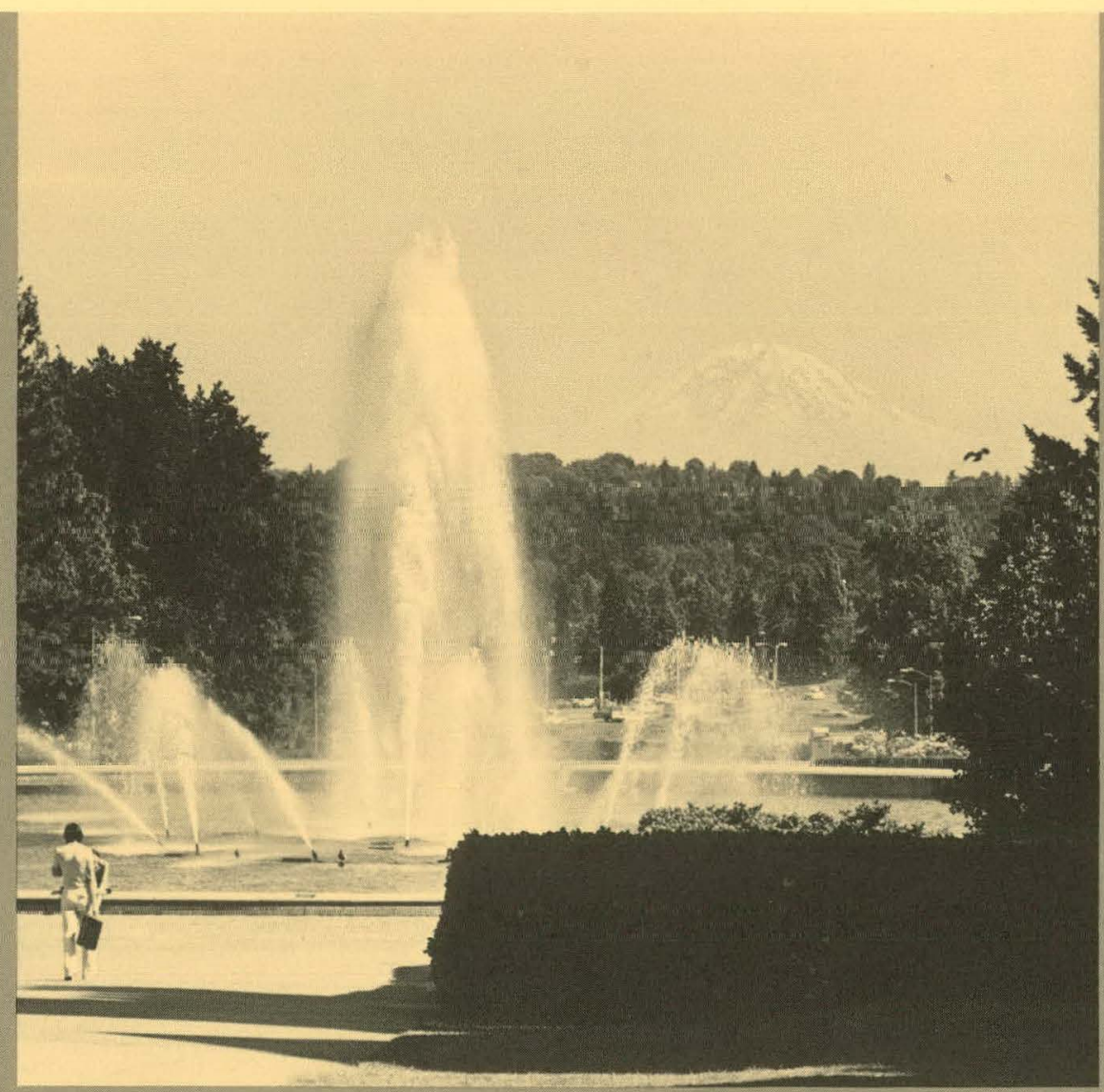




\section{DISCLAIMER}

This report was prepared as an account of work sponsored by an agency of the United States Government. Neither the United States Government nor any agency Thereof, nor any of their employees, makes any warranty, express or implied, or assumes any legal liability or responsibility for the accuracy, completeness, or usefulness of any information, apparatus, product, or process disclosed, or represents that its use would not infringe privately owned rights. Reference herein to any specific commercial product, process, or service by trade name, trademark, manufacturer, or otherwise does not necessarily constitute or imply its endorsement, recommendation, or favoring by the United States Government or any agency thereof. The views and opinions of authors expressed herein do not necessarily state or reflect those of the United States Government or any agency thereof. 


\section{DISCLAIMER}

Portions of this document may be illegible in electronic image products. Images are produced from the best available original document. 


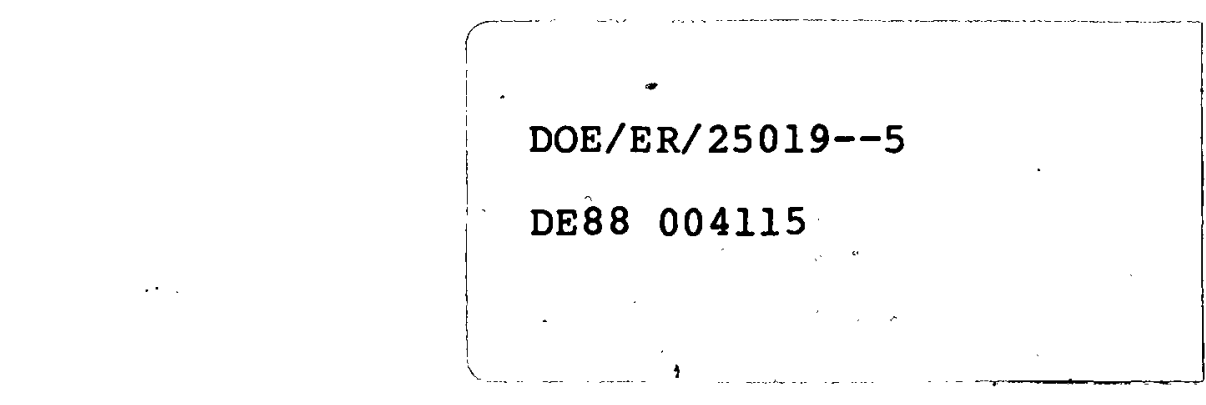

A Variational Principle For The Ackerberg-O'Malley Resonance Problem

R. Srinivasan

Technical Report No. $87-7$

August 1987

\section{DISCLAIMER}

This report was prepared as an account of work sponsored by an agency of the United States Government. Neither the United States Government nor any agency thereof, nor any of their employees, makes any warranty, express or implied, or assumes any legal liability or responsibility for the accuracy, completeness, or usefulness of any information, apparatus, product, or process disclosed, or represents that its use would not infringe privately owned rights. Reference herein to any specific commercial product, process, or service by trade name, trademark, manufacturer, or otherwise does not necessarily constitute or imply its endorsement, recommendation, or favoring by the United States Government or any agency thereof. The views and opinions of authors expressed herein do not necessarily state or reflect those of the United States Government or any agency thereof. 
A VARIATIONAL PRINCIPLE FOR THE
ACKERBERG-0'MALLEY RESONANCE PROBLEM*

R. Srinivasant

Key Words: Singular perturbation, resonance, variational principle, exact solutions

AMS (MOS) Subject Classifications: 34E15,20.

Abbreviated Title:

VARIATIONAL PRINCIPLE FOR ACKERBERG-O'MALLEY RESONANCE

* This research was supported by the U.S. Department of Energy under Grant No. DE-FG06-86ER25019.

† Department of Applied Mathematics, University of Washington, Seattle, Washington 98195. 


\section{Abstract}

A new variational principle is proposed for determining the asymptotic expansion of the solution of the Ackerberg-0'Malley resonance problem [Stud. Appl. Math., 49 (1970), pp. 277-295] to any order in $\varepsilon$. The method used differs from that originally proposed by Grasman and Matkowsky [SIAM J. Appl. Math., 32 (1977), pp. 588-597] in that variations are allowed in the boundary values of a transformed problem, making the calculation applicable to any order in $\varepsilon$. Explicit results using the new approach are reported to $O(\varepsilon)$ and confirmed with asymptotic expansions of the exact solution. The $O(1)$ results agree with those reported in the literature, and the $0(\varepsilon)$ results are new. It is not difficult to perform the higher order calculations using the new variational approach, in contrast to the current methods in use. In the case where the coefficient functions are analytic in the independent variable and $\varepsilon$; and the outer expansion satisfies a certain restriction, the exact solution presented provides an alternative and equally simple method for calculating the asymptotic expansion to any order in $\varepsilon$. 


\section{Introduction}

We consider once again the well known problem of Ackerberg and O'Malley [2]--the asymptotic solution as $\varepsilon+0^{+}$of the singular boundary value probiem

$$
\begin{aligned}
& \varepsilon y^{\prime \prime}-f(x, \varepsilon) y^{\prime}+g(x, \varepsilon) y=0 \\
& y(-1, \varepsilon)=\alpha(\varepsilon), \quad y(t, \varepsilon)=\beta(\varepsilon)
\end{aligned}
$$

where $0<a \leq t \leq b$, and $t$ is fixed as $\varepsilon+0^{+}$. Here $a(\varepsilon) ; \beta(\varepsilon)$, $f(x, \varepsilon), g(x, \varepsilon)$ are $C^{\infty}$ functions on $\varepsilon \in[0,1]$ and on $x \in[-1, b]$. We also assume $f(0,0)=0$ and $f^{\prime}(x, 0)=f_{x}(x, 0)>0$ on $[-1, b]$. Ackerberg and O'Malley [2] observed that this problem can have a nontrivial outer expansion only if

$$
\frac{g(0,0)}{f^{\prime}(0,0)}=n
$$

where $\mathrm{n}$ is a nonnegative integer. Assuming (1.2), the leading term of the outer expansion is

$$
y_{0}(x)=c_{0} k_{0}(x)
$$

where. $c_{0}$ is a constant, and

$$
k_{0}(x)=x^{n} \exp \int_{0}^{x}\left[\frac{g(s, 0)}{f(s, 0)}-\frac{n}{s}\right] d s .
$$

Because (1.2) is satisfied, $k_{0}(x) \in C^{\infty}[-1, b]$, and is, in particular, nonsingular at $x=0$. Matkowsky [8] proposed that, in order for (1.3a) to have a nonzero value of $c_{0}$, all the higher order terms of the outer expansion must be in $C^{\infty}[-1, b]$ as well. Thus, resonance can only occur (in the sense of Kopell [6]) if, in addition to (1.2), an infinite number of criteria are 
satisfied by $f(x, \varepsilon)$ and $g(x, \varepsilon)$ at $(x, \varepsilon)=(0,0)$. We refer to these, together with (1.2), as the Matkowsky criteria. Kopell [6] and later, Kreiss [7] showed that the Matkowsky criteria are necessary for resonance. In the case where $f(x, \varepsilon)$ and $g(x, \varepsilon)$ are analytic in, $x$ and $\varepsilon$ and satisfy certain other restrictions, the Matkowsky criteria are necessary and sufficient for resonance $[7,10]$. Kope11 [6] also showed that any nonresonant problem can be converted to a resonant one by adding to $g(x, \varepsilon)$ an appropriate $C^{\infty}[0,1]$ function of $\varepsilon$. In particular, it is possible to add certain exponentially small functions of $\varepsilon[9,6,11,13]$ (we indicate exponentially small terms by the notation $o\left(\varepsilon^{k}\right)$; it is understood that $k$ can assume any nonnegative integer value).

For problems which exhibit resonance, neither the location of the boundary layers nor the constants $c_{k}(k=0,1,2 \ldots)$ in the outer expansion are determined by the procedure of matched asymptotic expansions. One way to determine these constants is through a variational approach first proposed by Grasman and Matkowsky [4]. In their method, the true value of $c_{0}$ occurring in the : $O(1)$ uniform solution is postulated to be that which makes an appropriate functional (whose Euler-Lagrange equation is (1.1a)) stationary to variations in $c_{0}$. Williams [13] and Skinner [11] have shown that it is necessary to include the full outer expansion in the $0(1)$ uniform solution; in order for the variational principle to yield the correct result for $c_{0}$. In this paper, we propose a modified variational principle (sec. 2) that is easy to implement to any order in $\varepsilon$, and compare our method to that of. the Grasman-Matkowsky (GM) variational principle (sec. 3). We exhibit our results to $O(\varepsilon)$ for the general problem (1.1), in the case where the function $f$ and $g$ are analytic in $x$ and $\varepsilon$ (sec. 4). In sec. 5, this result is re-derived using the asymptotic expansion of the exact solution-- 
this method is also equally simple to implement to any order in $\varepsilon$. We also consider an example in sec. 5 where the function $g(x, \varepsilon)$ contains an exponentially small term in $\varepsilon$ and show by comparing with the asymptotic expansion of the exact solution that our method produces the correct results to $O(\varepsilon)$ at least.

\section{Variational Principle}

The uniformly valid solution of (1.1) to any algebraic order in $\varepsilon$ can be expressed as follows, using the standard technique of matched asymptotic expansions:

$$
y_{u}(x, \varepsilon)=\lambda(\varepsilon) z(x, \varepsilon)+v(x, \lambda(\varepsilon), \varepsilon)
$$

where

$$
\lambda(\varepsilon)=\sum_{k=0}^{\infty} \varepsilon^{k} c_{k}
$$

$$
z(x, \varepsilon)=k_{0}(x)\left(1+\sum_{j=1}^{\infty} \cdot \varepsilon^{j} k_{j}(x)\right)
$$

$$
\begin{aligned}
& v(x, \lambda(\varepsilon), \varepsilon)=(\alpha(\varepsilon)-\lambda(\varepsilon) z(-1, \varepsilon))\left(1+\sum_{k=1}^{\infty} \varepsilon^{k} p_{k}\left(x_{1}\right)\right) e^{f(-1,0) x_{1}} \\
& +(\beta(\varepsilon)-\lambda(\varepsilon) z(t, \varepsilon))\left(1+\sum_{k=1}^{\infty} \varepsilon^{k} q_{k}\left(x_{2}\right)\right) e^{-f(t, 0) x_{2}} .
\end{aligned}
$$

$$
x_{1}=\frac{x+1}{\varepsilon}, \quad x_{2}=\frac{t-x}{\varepsilon}
$$

$$
p_{k}(0)=0, \quad q_{k}(0)=0, k=1,2, \ldots .
$$

Here $\lambda(\varepsilon) z(x, \varepsilon)$ is the outer solution; $k_{j}(x), j=1,2, \ldots$ are integrals similar to $k_{0}(x)$ in $(1.3 b)$ such that $k_{0}(x) k_{j}(x)$ are in $c^{\infty}[-1, b]$ because of the Matkowsky criteria. $p_{k}$ and $q_{k}$ are certain polynomials satisfylng (2.1f) (which ensures that the boundary conditions 
(1.1b) are satisfied by $y_{u}$ ). Observe that all the undetermined constants occur only in the form $\lambda(\varepsilon)$ in (2.1b); thus, when considered as a function of $\varepsilon$, there is only one undetermined constant $\lambda(\varepsilon)$. That (2.1) is the correct form for $y_{u}$ can be verified directly; this is also clear from the exact solutions we consider in sec. 5 .

Make the following transformation in (1.1):

$$
y(x, \varepsilon)=z(X, \varepsilon) Y(X, \varepsilon) .
$$

The new variable $y$ satisfies

$$
\varepsilon Y^{\prime \prime}-F(X, \varepsilon) Y^{\prime}+G(X, \varepsilon) Y=0
$$

and boundary conditions

$$
Y(-1, \varepsilon)=\frac{\alpha(\varepsilon)}{Z(-1, \varepsilon)} \quad, \quad Y(t, \varepsilon)=\frac{\beta(\varepsilon)}{Z(t, \varepsilon)}
$$

where

$$
\begin{aligned}
& F(x, \varepsilon)=f(x, \varepsilon)-2 \varepsilon \frac{z^{\prime}(x, \varepsilon)}{z(x, \varepsilon)} \\
& G(x, \varepsilon)=\frac{R_{1}[z]}{z}:
\end{aligned}
$$

and $R_{1}[z]$ is the residual from (1.1a) given by

$$
R_{1}[z]=\varepsilon z^{\prime \prime}-f(x, \varepsilon) z^{\prime}+g(x, \varepsilon) z \text {. }
$$

The uniformly valid solution for $Y$ is (from (2.1), (2.2)):

$$
Y(x, \varepsilon)=\lambda(\varepsilon)+w(x, \lambda, \varepsilon)
$$

where

$$
w(x, \lambda, \varepsilon)=\left(\frac{\alpha(\varepsilon)}{z(-1, \varepsilon)}-\lambda(\varepsilon)\right)\left(1+\sum_{k=1}^{\infty} p_{k}\left(x_{1}\right)\right) e^{f(-1,0) x_{1}}
$$

$$
+\left(\frac{\beta(\varepsilon)}{z(t, \varepsilon)}-\lambda(\varepsilon)\left(1+\sum_{k=1}^{\infty} \varepsilon^{k} Q_{k}\left(x_{2}\right)\right) e^{-f(t, 0) x_{2}} .\right.
$$


$(2.6 c)$

$$
P_{k}(0)=0 \quad, \quad Q_{k}(0)=0 .
$$

The polynomials $P_{k}, Q_{k}$ are obtained by replacing $f$ and $g$ by $F$ and zero respectively in $p_{k}, q_{k}$. We assume that the Matkowsky criteria are satisfied and further, that $f$ and $g$ are such that the outer expansion $z(x, \varepsilon)$ is a power series in $\varepsilon$ that converges uniformly to a nontrivial solution of the analytic part of (1.1a). Note that our restriction on $z$ implies that resonance occurs in the sense of Kopell [6]. If $f$ and $g$ are analytic in $x, \varepsilon$, we must have $R_{1}[z]=0$. If $g(x, \varepsilon)$ has an additive exponentially small term in $\varepsilon$, we have $G(x, \varepsilon)=o\left(\varepsilon^{k}\right)$. These are the only situations considered in this paper, although our results for the analytic case may apply under less restrictive conditions if resonance occurs.

We postualte that the true value of $\lambda(\varepsilon)$ is determined by

$$
\left.\frac{d I}{d \lambda^{\star}}\right|_{\lambda^{\star}=\lambda(\varepsilon)}=0
$$

where

$$
I\left(\lambda^{\star}, \varepsilon\right)=\int_{-1}^{t} L\left(x, \tilde{Y}\left(x, \lambda^{*}, \varepsilon\right), \tilde{Y}^{\prime}, \varepsilon\right) d x
$$

and

$$
\tilde{Y}\left(x, \lambda^{\star}, \varepsilon\right)=(1-A) \lambda(\varepsilon)+A \lambda^{\star}+W\left(x, \lambda^{\star}, \varepsilon\right)
$$

Following Kevorkian and Cole [5], we chouse the Lagrangian as

$$
L\left(X, Y, Y^{\prime}, \varepsilon\right)=\left(\varepsilon Y^{\prime 2}-G(X, \varepsilon) Y^{2}\right) e^{-H(X, \varepsilon) / \varepsilon}
$$

where

$$
H(X, \varepsilon)=\int_{0}^{X} F(s, \varepsilon) d s
$$

In $(2.7 c), \lambda^{\star}$ is a parameter and $\lambda(\varepsilon)$, the exact value; the constant $A$ 
is determined later. Carrying through the differentiation in (2.7a), we obtain

$$
I_{1}(\lambda, \varepsilon)+I_{2}(\lambda, \varepsilon)-\left[\varepsilon W^{\prime}\left(A+\frac{\partial W}{\partial \lambda}\right) e^{-H(x, \varepsilon) / \varepsilon}\right]_{-1}^{t}=0
$$

where

$$
\begin{aligned}
& I_{1}(\lambda, \varepsilon)=\int_{-1}^{t} R_{2}[\lambda]\left(A+\frac{\partial W}{\partial \lambda}\right) e^{-H(x, \varepsilon) / \varepsilon} d x \\
& I_{2}(\lambda, \varepsilon)=\int_{-1}^{t} R_{2}[W]\left(A+\frac{\partial W}{\partial \lambda}\right) e^{-H(x, \varepsilon) / \varepsilon} d x
\end{aligned}
$$

and $R_{2}$ is the residual from (2.3a) defined by

$$
R_{2}[u]=\varepsilon u^{\prime \prime}-F(x, \varepsilon) u^{\prime}+G(x, \varepsilon) u .
$$

Using (2.6), it is easy to see that

$$
I_{1}(\lambda, \varepsilon)=A \lambda(\varepsilon) I_{3}(\varepsilon)+O\left(\varepsilon^{k}\left[e^{-h(-1,0) / \varepsilon}+e^{h(t, 0) / \varepsilon}\right]\right)
$$

$$
I_{2}(\lambda, \varepsilon)=O\left(\varepsilon^{k}\left[e^{-h(-1,0) / \varepsilon}+e^{-h(t, 0) / \varepsilon}\right]\right)
$$

$$
\left.\frac{\partial w}{\partial \lambda}\right|_{x=t}=-1+o\left(\varepsilon^{k}\right)=\left.\frac{\partial w}{\partial \lambda}\right|_{x=-1}
$$

where

$$
h(x, \varepsilon)=\int_{0}^{x} f(s, \varepsilon) d s
$$

$$
I_{3}(\varepsilon)=\int_{-1}^{t} G(x, \varepsilon) e^{-H(x, \varepsilon) / \varepsilon} d x
$$

Note in particular that $(2.9 \mathrm{~b})$ is a consequence of including an infinite number of terms in $w(x, \lambda, \varepsilon)$. We comment on this in the next section, where. our method is compared to the GM variational principle. Using (2.9) in (2.8a), we obtain 


$$
\left(\frac{A}{I-A}\right) \lambda(\varepsilon) I_{3}(\varepsilon)+\left[\varepsilon W^{\prime} e^{-H(x, \varepsilon) / \varepsilon}\right]_{-1}^{t}=o\left(\varepsilon^{k}\left[e^{-h(-1,0) / \varepsilon}+e^{-h(t, 0) / \varepsilon}\right]\right) .
$$

Equation (2.11) is our final result. The appropriate value of $A$ is detcrmined in sec. 5 .

3. Comparison to the GM Variational Principle $[4,11,12]$

The GM variational principle is equivalent to:

$$
\begin{aligned}
\lambda(\varepsilon) I_{4}(\varepsilon)+I_{5}(\lambda, \varepsilon)+O\left[\exp \left(f(-1,0) \frac{t+1}{\varepsilon}-\frac{h(-1,0)}{\varepsilon}\right)+\exp \left(-f(t, 0) \frac{t+1}{\varepsilon}\right.\right. & \left.\left.-\frac{h(t, 0)}{\varepsilon}\right)\right] \\
& =0
\end{aligned}
$$

where, using (2.1),

$$
\begin{aligned}
& I_{4}(\varepsilon)=\int_{-1}^{t} R_{1}[z] \frac{\partial y}{\partial \lambda} e^{-h(x, \varepsilon) / \varepsilon} d x \\
& I_{5}(\lambda, \varepsilon)=\int_{-1}^{t} R_{1}[v] \frac{\partial y}{\partial \lambda} e^{-h(x, \varepsilon) / \varepsilon} d x .
\end{aligned}
$$

In the analytic case, assuming $z(x, \varepsilon)$ is a convergent power series as before, we can set $R_{1}[z]=0$, and hence $I_{4}(\varepsilon)=0$. If an infinite number of terms are fincluded in $v(x, \lambda, \varepsilon)$ (given by $(2,1 d)$ ), we must have

$$
I_{5}(\lambda, \varepsilon)=o\left(\varepsilon^{k}\left[e^{-h(-1,0) / \varepsilon}+e^{-h(t, 0) / \varepsilon}\right]\right) .
$$

Equation (3.1d) is apparent from the observation that the coeffictent of $\varepsilon^{k} e^{-h(-1,0) / \varepsilon}$ or $\varepsilon^{k} e^{-h(t, 0) / \varepsilon}$ in $I_{5}(\lambda, \varepsilon)$ vanishes for all $k$. Thus, in general, it is possible that the end-point contribution given by the third term of (3.1a) dominates the first two terms. However, this gives incorrect results for $\lambda(\varepsilon)$, as can be easily verified. The correct interpretation of the GM 
variational principle requires that only the leading term of $v$ be taken in $I_{5}(\lambda, \varepsilon)$, and the evaluation of $\lambda(\varepsilon)$ be restricted to $O(1)$. Note that in this case, (3.1) yields a correction of $O\left(\varepsilon^{1 / 2}\right)$ to the leading term of $\lambda(\varepsilon)$ which clearly implies that the GM method is not applicable beyond $0(1)$.

In our method, where we apply a modification of the original idea of ref. [4] to the transformed equation (2.3), we allow variations in the boundary values of $Y$. Thus, the end-point contributions become significant; in the analytic case, with $G(x, \varepsilon)=I_{3}(\varepsilon)=0$, only the end-point contributions survive in (2.11). This has the interpretation of the 'natural boundary condition" that results from the Euler-Lagrange technique. Note that the value of the parameter $A$ does not influence (2.11) in the analytic case--we only need $A \neq 1$ in order to introduce end-point contributions. However, only one particular value of $A$ produces the correct result in the nonanalytic case, as example 2 of sec. 5 demonstrates. To $O(1)$, this value of A makes our method entireily equivalent to the GM variational principle. The main advantage of eq. (2.11) is that it is very easy to implement to any order in $\varepsilon$.

4. The Analytic Case: Results to $O(\varepsilon)$

Assume that $f(x, \varepsilon), g(x, \varepsilon)$ are analytic in $x$ and $\varepsilon$ in addition to satisfying the Matkowsky criteria, and, because $z$ is assumed to be a. convergent power series in $\varepsilon$, we have $G(x, \varepsilon)=I_{3}(\varepsilon)=0$ in (2.11). Note that this is true if $z(x, \varepsilon)$ terminates (which happens, for example if $f(x, \varepsilon)=x$ and $g(x, \varepsilon)=n$, [2] or if $f(x, \varepsilon)=x, g(x, \varepsilon)=1-\varepsilon+x$ [12]). Equation (2.11) becomes

$$
\left[\varepsilon W^{\prime} e^{-H(x, \varepsilon) / \varepsilon}\right]_{-1}^{t}=O\left[\varepsilon^{k}\left(e^{-h(t, 0) / \varepsilon}+e^{-h(-1,0) / \varepsilon}\right)\right] .
$$


The uniformly valid solution for $Y$, as given in $(2.6)$, is specified to $O(\varepsilon)$ by

(4.2a)

$$
P_{1}\left(x_{1}\right)=f^{\prime}(-1,0) \frac{x_{1}^{2}}{2}-\psi(-1) x_{1}
$$

$$
Q_{1}\left(x_{2}\right)=f^{\prime}(t, 0) \frac{x^{2}}{2}+\psi(t) x_{2}
$$

where

$$
\psi(s)=\frac{f^{\prime}(s, 0)}{f(s, 0)}-f_{\varepsilon}(s, 0)+\frac{2 g(s, 0)}{f(s, 0)} .
$$

To evaluate $z(x, \varepsilon)$ to $0(\varepsilon)$ (eq. $(2.1 c\rangle)$, we need

$$
k_{1}(x)=\int \frac{x g_{0}^{2}+f_{0} g_{0}^{1}-g_{0} f_{0}^{\prime}-f_{\varepsilon}(s, 0) g_{0} f_{0}+g_{\varepsilon}(s, 0) f_{0}^{2}}{f_{0}^{3}} d s
$$

where

$$
g_{0}=g(s, 0), \quad f_{0}=f(s, 0),
$$

and $k_{0}(x) k_{1}(x)$ is in $c^{\infty}[-1, b]$ because of the Matkowsky criteria. Note that

$$
e^{-H(x, 0) / \varepsilon}=e^{-h(x, 0) / \varepsilon} u(x) k_{0}^{2}(x)\left(1-\frac{\varepsilon}{2} h_{\varepsilon \varepsilon}(x, 0)+2 \varepsilon k_{1}(x)+O\left(\varepsilon^{2}\right)\right)
$$

where

$$
u(x)=\exp \left[-h_{\varepsilon}(x, 0)\right] .
$$

Using (4.2) $-(4.4)$ and $(2.6)$ to evaluate (4.1) results in

$$
\begin{gathered}
f(t, 0) u(t) k_{0}(t) e^{-h(t, 0) / \varepsilon}\left[\left(\beta(0)-\lambda(\varepsilon) k_{0}(t)\right)(1-\varepsilon \omega(t))+\varepsilon B^{\prime}(0)-\varepsilon \beta(0) k_{1}(t)\right. \\
\left.+0\left(\varepsilon^{2}\right)\right]-f(-1,0) u(-1) k_{0}(-1) e^{-h(-1,0) / \varepsilon}\left[\left(\alpha(0)-\lambda(\varepsilon) k_{0}(-1)\right)(1-\varepsilon \omega(-1))\right. \\
\left.+\varepsilon \alpha^{\prime}(0)-\varepsilon \alpha(0) k_{1}(-1)+0\left(\varepsilon^{2}\right)\right]=0
\end{gathered}
$$


where

$(4.5 b)$

$$
\omega(s)=\frac{\psi(s)}{f(s, 0)}+\frac{1}{2} h_{\varepsilon \varepsilon}(s, 0)-2 k_{1}(s)
$$

Setting $\lambda(\varepsilon)=C_{0}+\varepsilon C_{1}+O\left(\varepsilon^{2}\right)$ in (4.5) results in the following values for $c_{0}, c_{1}:$

Case (i): $\quad h(t, 0)<h(-1,0)$

(4.6a) $\quad c_{0}=\frac{B(0)}{k_{0}(t)}$

(4.6b) $\quad c_{1}=\frac{\beta^{\prime}(0)}{k_{0}(t)}-c_{0} k_{1}(t)$

Case(ii): $\quad h(t, 0)>h(-1,0)$

(4.7a) $\quad c_{0}=\frac{\alpha(0)}{k_{0}(-1)}$

(4.7b) $\quad c_{1}=\frac{\alpha^{\prime}(0)}{k_{0}(-1)}-c_{0} k_{1}(-1)$.

Case (iii): $\quad h(t, 0)=h(-1,0)$

Let

$$
\begin{aligned}
& \mu=\frac{k_{0}(-1) f(-1,0) u(-1)}{k_{0}(t) f(t, 0) u(t)} \\
& \gamma=\mu k_{0}(-1)-k_{0}(t) .
\end{aligned}
$$

We obtain

$(4.8 \mathrm{a})$

$$
c_{0}=\frac{\mu \alpha(0)-B(0)}{\gamma}
$$

$$
\begin{aligned}
c_{1}= & {\left[\mu \alpha^{\prime}(0)-B^{\prime}(0)-\mu \alpha(0) k_{1}(-1)+B(0) k_{1}(t)\right] / \gamma } \\
& +\left[\left(B(0)-c_{0} k_{0}(t)\right) \omega(t)-\left(\alpha(0)-c_{0} k_{0}(-1)\right) \mu(1)(-1)\right] / \gamma .
\end{aligned}
$$


The results for $c_{0}$ are in agreement with $[4,11,14]$. In the next section we confirm the validity of the above results from the exact solution and consider the non-analytic case.

\section{Exact Solutions and the Non-Analytic Case}

Note that the restriction on $z$ at the beginning of sec. 4 implies the following equation for $Y$, with $G(x, \varepsilon)=0$ in (2.3) in the analytic case:

$$
\begin{aligned}
& \varepsilon Y^{\prime \prime}-F(X, \varepsilon) Y^{\prime}=0 \\
& Y(-1, \varepsilon)=\delta(\varepsilon) \quad, Y(t, \varepsilon)=\tau(\varepsilon)
\end{aligned}
$$

where

$$
\delta(\varepsilon)=\frac{a(\varepsilon)}{Z(-1, \varepsilon)} \quad \tau(\varepsilon)=\frac{\beta(\varepsilon)}{Z(t, \varepsilon)} .
$$

The exact solution of $(5.1)$ is.

$$
Y(x, \varepsilon)=\left(\frac{\tau(\varepsilon)-\delta(\varepsilon)}{J(t, \varepsilon)-J(-1, \varepsilon)}\right) J(x ; \varepsilon)+\lambda(\varepsilon)
$$

where

$$
J(x, \varepsilon)=\int_{0}^{x} e^{H(s, \varepsilon) / \varepsilon} d s
$$

$$
\lambda(\varepsilon)=\frac{\delta(\varepsilon)[J(-1, \varepsilon)]^{-1}-\tau(\varepsilon)[J(t, \varepsilon)]^{-1}}{[J(-1, \varepsilon)]^{-1}-[J(t, \varepsilon)]^{-1}} .
$$

The first term of (5.2a) corresponds to the boundary layers, and (5.3) is the exact expression for $\lambda(\varepsilon)$. To evaluate the asymptotic expansion of $\lambda(\varepsilon)$, note that near $r=t$

$$
\begin{aligned}
e^{H(r, \varepsilon) / \varepsilon}= & \frac{e^{h(t, 0) / \varepsilon}}{u(t) k_{0}^{2}(t)}\left[1+\varepsilon\left\{\frac{h_{\varepsilon \varepsilon}(t, 0)}{2}-2 k_{1}(t)+\frac{r_{2}^{2}}{2} f^{\prime}(t, 0)\right.\right. \\
& \left.\left.-r_{2}\left(f_{\varepsilon}(t ; 0)-\frac{2 g(t, 0)}{f(t, 0)}\right)\right\}+0\left(\varepsilon^{2}\right)\right] e^{-f(t, 0) r_{2}}
\end{aligned}
$$


where

$$
r_{2}=\frac{t-r}{\varepsilon}
$$

Inserting (5.4) into (5.2b) results in

$$
[J(t, \varepsilon)]^{-1}=\frac{e}{\varepsilon}^{-h(t, 0) / \varepsilon} u(t) k_{0}^{2}(t) f(t, 0)\left[1-\varepsilon \omega(t)+0\left(\varepsilon^{2}\right)\right] .
$$

Using (5.5), a similar expansion for $[J(-1, \varepsilon)]^{-1}$ and suitable expansions for $\delta(\varepsilon)$ and $\tau(\varepsilon)$ in (5.3) results in precisely $(4.6)-(4.8)$. Observe that these equations for $c_{0}$ and $c_{1}$ are invariant under the following changes:

(i) First set $k_{0}=1, k_{1}=0, g=0$ in (4.6)-(4.8).

(ii) Next replace $f(x, \varepsilon), h(x, \varepsilon), \alpha(\varepsilon), \beta(\varepsilon)$ by $F(x, \varepsilon), H(x, \varepsilon)$, $\delta(\varepsilon), \tau(\varepsilon)$ respectively.

This provides a convenient check on the validity of these equations. As a final example, consider

$$
\begin{aligned}
& \varepsilon y^{\prime \prime}-x y^{\prime}+\nu(\varepsilon) y=0 \\
& y(-1, \varepsilon)=\alpha(\varepsilon), \quad y(t, \varepsilon)=\beta(\varepsilon) .
\end{aligned}
$$

The exact solution of $(5.6)$ is

$$
y(X, \varepsilon)=e^{X^{2} / 4}\left[\varepsilon^{\nu / 2} \lambda(\varepsilon) D_{\nu}(X)+B(\varepsilon) D_{-\nu-1}(i X)\right]
$$

where

$$
x=x / \varepsilon^{1 / 2} \text {, and }
$$

$$
\varepsilon^{\nu / 2} \lambda(\varepsilon)=\left[\alpha(\varepsilon) e^{-1 / 4 \varepsilon_{D}}-\nu-1\left(\frac{i t}{\varepsilon^{1 / 2}}\right)-\beta(\varepsilon) e^{-t^{2} / 4 \varepsilon_{D}}{ }_{-\nu-1}\left(\frac{-i}{\varepsilon^{1 / 2}}\right)\right] / K(t, \varepsilon)
$$


(5.7C) $\quad B(\varepsilon)=\left[B(\varepsilon) e^{-t^{2} / 4 \varepsilon_{D}}\left(-\frac{1}{\varepsilon^{1 / 2}}\right)-\alpha(\varepsilon) e^{-1 / 4 \varepsilon_{D_{\nu}}}\left(\frac{t}{\varepsilon^{1 / 2}}\right)\right] / K(t, \varepsilon)$

where

(5.7d) $\quad K(t, \varepsilon)=D_{\nu}\left(-\frac{1}{\varepsilon^{1 / 2}}\right) D_{-\nu-1}\left(\frac{i t}{\varepsilon^{1 / 2}}\right)-D_{\nu}\left(\frac{t}{\varepsilon^{1 / 2}}\right) D_{-\nu-1}\left(-\frac{i}{\varepsilon^{1 / 2}}\right)$.

$D_{\nu}$ is the parabolic cylinder function. From [3], the following expansions apply for $|x| \gg \varepsilon^{1 / 2}$ :

$$
D_{\nu}(x)=x^{\nu} \varepsilon-\frac{\nu}{2} \mathrm{e}^{-x^{2} / 4}\left(1-\varepsilon \frac{\nu(\nu-1)}{2 x^{2}}+O\left(\varepsilon^{2}\right)\right),
$$

$$
X \rightarrow+w
$$

$$
\begin{gathered}
D_{\nu}(-x)=e^{i \pi \nu} D_{\nu}(x)+x^{-\nu-1} e^{x^{2} / 4} \sqrt{2 \pi}[r(-\nu)]^{-1}\left(1+\frac{\varepsilon(\nu+1)(\nu+2)}{2 x^{2}}+o\left(\varepsilon^{2}\right)\right) \\
x \rightarrow+\infty \\
D_{-\nu-1 .}(i x)=(i x)^{-\nu-1} \varepsilon(\nu+1) / 2 e^{x^{2} / 4}\left(1+\frac{(\nu+1)(\nu+2)}{2 x^{2}}+0\left(\varepsilon^{2}\right)\right) \\
x \rightarrow \pm \infty
\end{gathered}
$$

where $x=x / \varepsilon^{1 / 2}$. If $v=n$, we have $[r(-v)]^{-1}=0$, and using (5.8)

in (5.7b) reproduces the results from eqs. $(4.6)-(4.8)$. Consider the example of $[11]$ :

$$
\nu(\varepsilon)=1-\left(2 \pi \varepsilon^{3}\right)^{-1 / 2} e^{-d^{2} / 2 \varepsilon}
$$

where $d>0$. From $[1]^{\circ}$,

$$
\text { . }[\Gamma(-v)]^{-1}=-(1-v(\varepsilon))+O\left([1-\nu]^{2}\right), \quad \nu+1^{-}
$$

Using $(5.8)-(5.10)$ in $(5.7 b)$ results in

$$
\lambda(\varepsilon)=\frac{W(t, \varepsilon)}{E(t, \varepsilon)}
$$


where

(5.11b) $W(t, \varepsilon)=\left[B(0)\left(1-\frac{3 \varepsilon}{t^{2}}\right)+\varepsilon B^{\prime}(0)+0\left(\varepsilon^{2}\right)\right] t^{2} e^{-t^{2} / 2 \varepsilon}-[\alpha(0)(1-3 \varepsilon)$

$$
\left.+\varepsilon \alpha(0)+0\left(\varepsilon^{2}\right)\right] e^{-1 / 2 \varepsilon}
$$

(5.11c) $E(t, \varepsilon)=t^{3}\left(1-\frac{3 \varepsilon}{t^{2}}+0\left(\varepsilon^{2}\right)\right) e^{-t^{2} / 2 \varepsilon}+\left(1-3 \varepsilon+0\left(\varepsilon^{2}\right)\right) e^{-1 / 2 \varepsilon}+e^{-d^{2} / 2 \varepsilon}$.

This leads to the following values for $c_{0}, c_{1}$ :

Case 1: $d>1$ and/or $d>t$

$\lambda(\varepsilon)$ is same as for $\nu=1$ (eqs. $(4.6)-(4.8)$ ).

Case 2: $0<d<1$

$$
\begin{aligned}
\lambda(\varepsilon) & =o\left(\varepsilon^{k}\right), \quad d<t \\
& =\frac{B(0) t^{2}}{i+t^{3}}+\varepsilon\left[\frac{\beta^{\prime}(0) t^{2}}{1+t^{3}}-\frac{3 B(0)}{\left(1+t^{3}\right)^{2}}\right]+0\left(\varepsilon^{2}\right), \quad d=t .
\end{aligned}
$$

Case $3: d=1$

$$
\begin{aligned}
\lambda(\varepsilon) & =-\frac{\alpha(0)}{2}+\varepsilon\left(-\frac{\alpha^{\prime}(0)}{2}+\frac{3}{4} \alpha(0)\right)+0\left(\varepsilon^{2}\right), & & t>1 \\
& =\frac{\beta(0)-\alpha(0)}{3}+\varepsilon\left(\frac{\beta^{\prime}(0)-\alpha^{\prime}(0)}{3}+\frac{\alpha(0)-\beta(0)}{3}\right)+0\left(\varepsilon^{2}\right), & & t=1 .
\end{aligned}
$$

The 0 (1) results agree with those of Skinner [11]. A straightforward calculation shows that the asymptotic expansion of (5.7a) reduces to the form (2.1) in all of the above cases. Using equation (2.11) for this example, with

$$
G(x, \varepsilon)=-\left(2 \pi \varepsilon^{3}\right)^{-1 / 2} \cdot \mathrm{e}^{-d^{2} / 2 \varepsilon}
$$


and hence

$$
I_{3}(\varepsilon)=-e^{-d^{2} / 2 \varepsilon}+o\left(\varepsilon^{k} e^{-d^{2} / 2 \varepsilon}\right)
$$

results precisely in $(5.11)$ and $(5.12)$ if

$$
A=\frac{1}{2}
$$

Equation (5.14) determines the value of $A$ needed in (2.11) to produce the correct result to at least $O(\varepsilon)$.

\section{Concluding Remarks}

In the non-analytic case, it is worth considering other examples and going to higher orders to see if (2.11) and (5.14) still apply, although we have not done so here. It is clear that (2.11) is valid in the analytic case if $z(x, \varepsilon)$ satisfies the restriction mentioned in sec. 2. In this case (5.3) is the exact expression for $\lambda(\varepsilon)$. It is possible that (5.3) and the results of sec. 4 are valid even without the above restriction in the analytic case, if resonance occurs. Our results for $c_{1}$ are new and it is not difficult to evaluate (2.11) to higher orders in $\varepsilon$, in contrast to the current methods in use.

\section{Acknowledgment}

The author is grateful to Professor J. Kevorkian for an excellent introduction to perturbation techniques through two graduate courses. The ". research presented in this paper was inspired by one of Professor Kevorkian's lectures: The author gratefully acknowledges generous financial support from the Department of Applied Mathematics at the University of Washington. 


\section{REFERENCES}

[1] Abramowitz and Stegun, Handbook of Mathematical Functions, Dover ', Publications, New York, 1965.

[2] R.C. Ackerberg and R.E. O'Malley, Jr., Boundary layer problems exhibiting resonance, Stud. Appl. Math., 49 (1970), pp. 277-295.

[3] C.M. Bender and S.A. Orszag, Advanced Mathematical Methods for Scientists and Engineer's, McGraw Hill, New York, 1978.

[4] J. Grasman and B.J. Matkowsky, A variational approach to singularly perturbed boundary value problems for ordinary and partial

differential equations with turning points, this Journal, 32 (1977), pp. 588-597.

[5] J. Kevorkian and J.D. Cole, Perturbation Methods in Applied Mathematics, Springer-Verlag, New York, 1981.

[6] N. Kopell, A geometric approach to boundary layer problems exhibiting resonance, this Journal, 37 (1979), pp. 436-458.

[7] H.0. Kreiss, Resonance for singular perturbation problems, this Journal, 41 (1981), pp. 331-344.

[8] B.J. Matkowsky, on boundary iayer problems exhibiting resonance, SIAM Rev., 17 (1975), pp. 82-100; Errata, SIAM Rev., 18 (1976), p. 112.

[9] F.W.J. Olver, Sufficient conditions for Ackerberg-O'Malley resonance, SIAM. J. Math. Anal., 9 (1978), pp. 328-355.

[10] Y. Sibuya, A theorem concerning uniform simplification at a transition point and the problem of resonance, MRC Technical Summary Report No. 2103 .

[11] L.A. Skinner, Uniform solution of boundary Zayer problems exhibiting resonance, this Journal, 47 (1987), pp. 225-231.

[12] A.M. Watts, A singular perturbation problem with a turning point, Bu11. Austral. Math. Soc., 5 (1971), pp. 61-73.

[13] Michael Williams, Another Look at Ackerberg-O'Malley resonance, this Journa , 11 (1981), pp. 288-293.

[14] E. Zauderer, Boundary value problems for a second order differential equation with a turning point, Studies in Appl. Math., 51 (1972), pp. 411-413. 